HZPP-9809

Dec. 10,1998

\title{
LEVY STABILITY INDEX FROM MULTIFRACTAL SPECTRUM
}

\author{
Hu Yuan Yu Meiling Liu Lianshou \\ Institute of Particle Physics, Huazhong Normal University, Wuhan 430079 China \\ Tel: 02787673313 \\ FAX: 02787662646 \\ email: liuls@iopp.ccnu.edu.cn
}

\begin{abstract}
A method for extracting the Levy stability index $\mu$ from the multi-fractal spectrum $f(\alpha)$ in high energy multiparticle production is proposed. This index is an important parameter, characterizing the non-linear behaviour of dynamical fluctuations in high energy collisions. Using the random cascading $\alpha$ model as example, the validity of this method is tested. It is shown that this method, basing on a linear fit, is consistent with and more accurate than the usual method of fitting the ratio of $q$ th to 2nd order multi-fractal (Rényi) dimensions to the Peschanski formula.
\end{abstract}

PACS number: $13.85 \mathrm{Hd}$

Keywords: Multiparticle production, Levy stability, Multifractal spectrum, Rényi dimension

\footnotetext{
${ }^{1}$ This work is supported in part by the National Natural Science Foundation of China. (NSFC) under Grant No.19575021.
} 
Recently, the search, starting from eighties ${ }^{[1]}$, for the non-linear phenomena — intermittency or fractality in the multiparticle final states of high energy collisions ${ }^{[2]}$ has had breakthrough $^{[3]}$. It is found that dynamical fluctuations do exist in high energy hadronhadron collisions, but is aniosotropic rather than isotropic ${ }^{[4]}$. Power law in the higherdimensional factorial moment versus partition number has been observed in hadron-hadron collisions, when the anisotropy of dynamical fluctuations is taken into account properly ${ }^{[5,6]}$. On the other hand, the dynamical fluctuations in $\mathrm{e}^{+} \mathrm{e}^{-}$collisions are found to be approximately isotropic ${ }^{[7]}$.

As a (self-affine or self-similar) fractal system, the multiparticle final state in high energy collisions can be characterised by an important parameter - the Levy stability index $\mu^{[8]}$. This parameter tells us the behaviour of elementary fluctuations at the tail of distribution. To extract its value from exprimental data more reliably is an essential task for the understanding of fluctuation dynamics.

Previously, people usually use the anomalous scaling of factorial moment ${ }^{[9]}$

$$
F_{q}=\frac{1}{M} \sum_{m=1}^{M} \frac{\left\langle n_{m}\left(n_{m}-1\right) \cdots\left(n_{m}-q+1\right)\right\rangle}{\left\langle n_{m}\right\rangle^{q}}
$$

to get the scaling index (IM index) $\phi_{q}$ and multi-fractal (Rényi) dimensions ${ }^{[10]}$

$$
D_{q}=1-\frac{\phi_{q}}{q-1}
$$

of integer order $q$, and then fit the ratio $\left(1-D_{q}\right) /\left(1-D_{2}\right)$ to the formula ${ }^{[11]}$

$$
\frac{1-D_{q}}{1-D_{2}}=\frac{1}{q-1} \frac{q^{\mu}-q}{2^{\mu}-2}
$$

to get the value of $\mu$. In practice only a few indices $\phi_{q}$ of integer $q>2(q=3,4,5$ e.g.) can be obtained and the resulting value of $\mu$ basing on a fit of very few points to the complicated formula (3) is obviously unsatisfactory.

In order to establish an alternative way for extracting the value of $\mu$, let us notice that the $q$ th order multi-fractal Rényi dimension $D_{q}$ of any order $q$ can be obtained from the multifractal spectrum $f(\alpha)$ through ${ }^{[10]}$

$$
D_{q}=\frac{1}{q-1}(q \alpha-f(\alpha))
$$

Therefore, it should be able to get the Levy stability index $\mu$ directly from the multifractal spectrum $f(\alpha)$. This short note is aimed to discuss this problem.

It can be seen from Eq.(4) that

$$
\tau_{q}=(q-1) D_{q}
$$

is the Legandre transform of $f(\alpha)$. Therefore, we have ${ }^{[12]}$

$$
\alpha_{q}=\frac{d \tau_{q}}{d q}
$$




$$
f(\alpha)=q \alpha_{q}-\tau_{q}
$$

From Eq.(3) we have

$$
D_{q}=1-A \frac{q^{\mu}-q}{q-1}
$$

where $A=\left(1-D_{2}\right) /\left(2^{\mu}-2\right)$. Inserting into Eq.(5) and making use of Eq's. (6),(7) we get

$$
\begin{gathered}
\tau_{q}=-1+(1+A) q-A q^{\mu}, \\
\alpha_{q}=1+A-\mu A q^{\mu-1}, \\
f(\alpha)=1-(\mu-1) A q^{\mu} .
\end{gathered}
$$

Therefore, defining

$$
B=1+A=1+\frac{1-D_{2}}{2^{\mu}-2}
$$

we finally get

$$
1-f(\alpha) \propto(B-\alpha)^{\mu /(\mu-1)} \quad \text { when } \alpha<B .
$$

Thus we propose the following procedure for getting the Levy stability index $\mu$ from the multi-fractal spectrum $f(\alpha)$ :

(1) Find out the value of $\alpha$ where $f(\alpha)=1$, call it $B$.

(2) Fit the $\alpha<B$ part of $\ln (1-f(\alpha))$ versus $\ln (B-\alpha)$ to a straight line and get the slope $C$.

(3) Get Levy stability index as

$$
\mu=\frac{C}{C-1} .
$$

As an example, we apply this method to the random cascading $\alpha$-model ${ }^{[13]}$. This model describes each multiparticle event as a series of steps, in which the initial phase space region $\Delta$ is repeatedly divided into $\lambda=2$ parts. After $\nu$ steps we get $M=2^{\nu}$ sub-cells of size $\delta=\Delta / M$. At each step $s$ the normalized particle density is obtained in each of the two parts by multiplication of the normalized density in the step $s-1$ by a particular value of the random variable $\omega_{\nu j_{\nu}}$, where $j_{\nu}$ is the position of a sub-cell at the $\nu$ th step $\left(1 \leq j_{\nu} \leq\right.$ $\left.2^{\nu}\right)$. The elementary fluctuation probability $\omega$ is chosen as ${ }^{[13]}$

$$
\omega_{\nu, 2 j-1}=\frac{1}{2}(1+\alpha r) \quad ; \quad \omega_{\nu, 2 j}=\frac{1}{2}(1-\alpha r),
$$

in which, $r$ is a random number distributed uniformly in the interval $[-1,1], \alpha$ is a model parameter, characterizing the strength of fluctuations. In our calculation, we have chosen its value as $\alpha=0.3$.

The resulting multi-fractal spectrum $f(\alpha)$ in this model are shown in Fig. 1. The value of $\alpha$ for $f(\alpha)=1$ is found to be $B=1.0113$. The fitting of $\left(1-D_{q}\right) /\left(1-D_{2}\right)$ to Eq.(3) and the linear fit of $\ln (1-f(\alpha))$ versus $\ln (B-\alpha)$ are shown in Fig's.2 and 3 respectively. The resulting Levy indices $\mu$ from these two methods with different regions of $q$ together with the corresponding $\chi^{2} / \mathrm{DF}$ are 
Fit from $\left(1-D_{q}\right) /\left(1-D_{2}\right)$

$$
(q=2,3,4,5) \quad \mu=1.929 \pm 0.059 \quad \chi^{2} / \mathrm{DF}=0.016 / 2 .
$$

Linear fit of $\ln (1-f(\alpha))$ versus $\ln (B-\alpha)$

$$
\begin{array}{llll}
(q=0.1-5, & \alpha=1.009-0.909) & \mu=1.962 \pm 0.013 & \chi^{2} / \mathrm{DF}=0.758 / 48 \\
(q=2-5, & \alpha=0.967-0.909) & \mu=1.950 \pm 0.022 & \chi^{2} / \mathrm{DF}=0.107 / 29 . \\
(q=3-5, & \alpha=0.945-0.909) & \mu=1.940 \pm 0.015 & \chi^{2} / \mathrm{DF}=0.015 / 19
\end{array}
$$

cf. Fig.4.

Thus we see that the method we proposed for extracting the Levy stability index $\mu$ from the multi-fractal spectrum $f(\alpha)$, basing on a linear fit, is consistent with the usual method of fitting the ratio of $q$ th to 2nd order multi-fractal (Rényi) dimensions to the Peschanski formula Eq.(3). It is more accurate and reliable and is reasonably stable with respect to the region of $q$ or $\alpha$ used in fitting.

This method can readily be applied to real experimental data analysis as a better way for getting Levy stability index.

\section{Acknowledgement}

The authors thank Fu Jinghua and Liu Feng for helpful discussions. 


\section{References}

[1] Burnett T H et al. Phys. Rev. Lett., 1983, 50: 2062.

[2] See for example the review article: De Wolf E A, I. M. Dremin , W. Kittel, Phys. Rep. 270 (1996) 1.

[3] Liu Lianshou. Talk given at the 28th International Conference on High Energy Physics, Vancouver, 1998.

[4] Wu Yuanfang and Liu Lianshou, Phys. Rev. Lett. 70 (1993) 3197.

[5] N M Agababyan et al. (NA22), Phys. Lett. B382 (1995) 305; B431 (1988) 451.

[6] S. Wang, Z. Wang and C. Wu, Phys. Lett. B410 (1997) 323.

[7] Liu Fuming, Liu Feng and Liu Lianshou, On the dynamical fluctuations in the multiparticle final states in $e^{+} e^{-}$collisions, HE\&N Phys. to be published.

[8] A Y Khintchine and P Levy, Sur Les Lois Stables, C. R. Acad. Sci. (Paris), 202 (1936) 374.

[9] A. Białas and R. Peschanski, Nucl. Phys. B273 (1986) 703; B308 (1988) 857.

[10] G. Paladin and A. Vulpiani, Phys. Rep. 156 (1987) 147.

[11] Ph. Braxa and R. Peschanski, Phys. Lett. B253 (1991) 315.

[12] R. C. Hwa, Phys. Rev. D51 (1995) 3323.

[13] Wu Yuanfang, Zhang Kunshi and Liu Lianshou, Chinese Science Bulletin 36 (1991) 1077.

\section{Figure Captions}

Fig.1 Multi-fractal spectrum

Fig.2 Fitting of $\left(1-D_{q}\right) /\left(1-D_{2}\right)$ to Eq.(3) for $q=2,3,4,5$

Fig.3 Linear fit of $\ln (1-f(\alpha))$ vs. $\ln (B-\alpha)$ for $q=0.1-5$

Fig.4 The resulting $\mu$ value from different ways of fitting together with the $q$ region used and the $\chi^{2} / \mathrm{DF}$ 


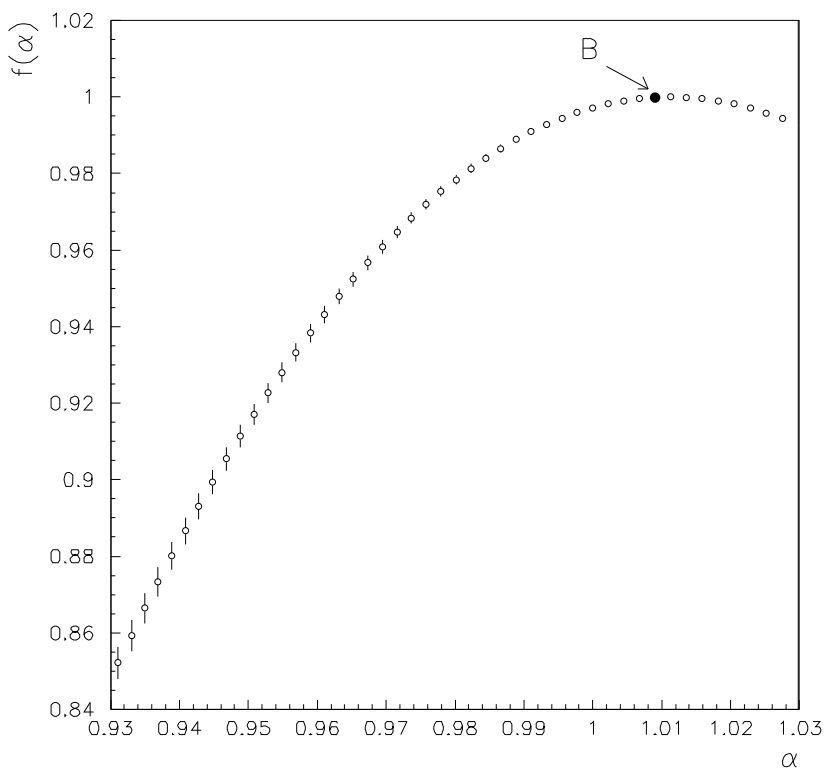

Fig.1 Multi-fractal spectrum

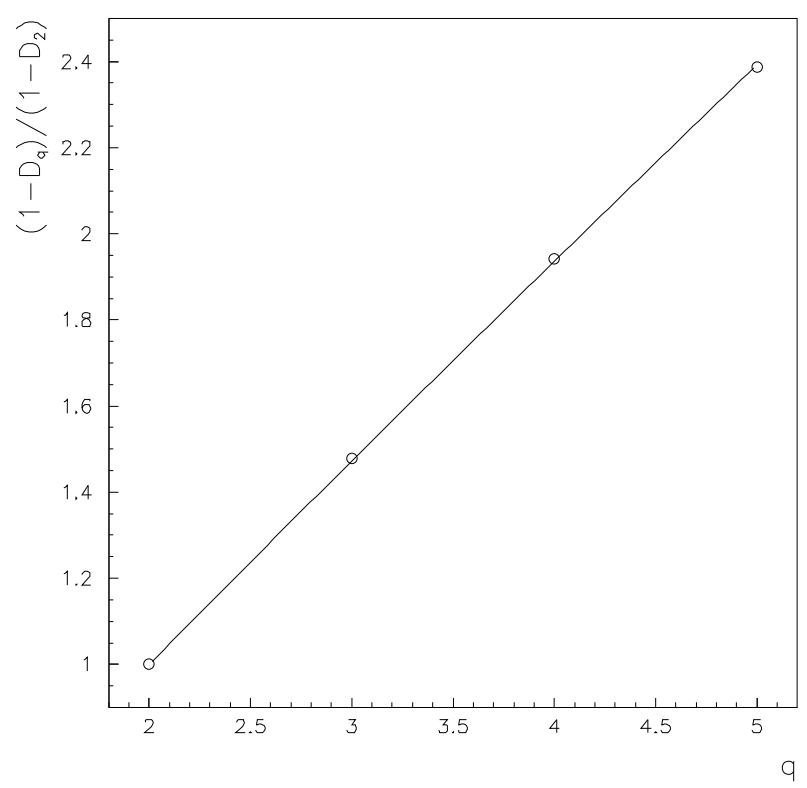

Fig.2 Fitting of $\left(1-D_{q}\right) /\left(1-D_{2}\right)$ to Eq.(3) for $q=2,3,4,5$ 


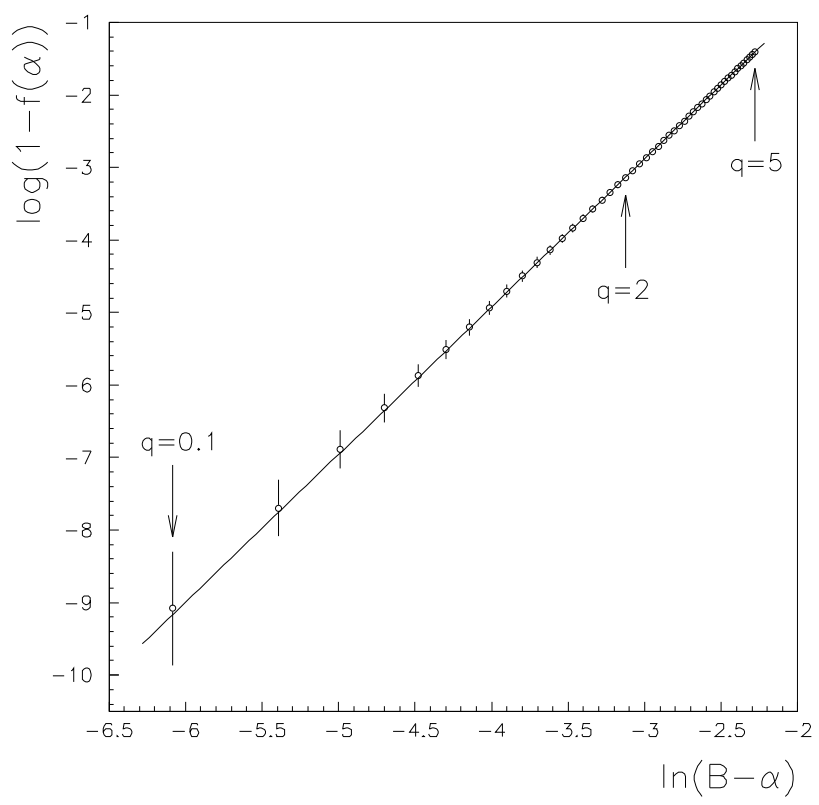

Fig.3 Linear fit of $\ln (1-f(\alpha))$ vs. $\ln (B-\alpha)$ for $q=0.1-5$

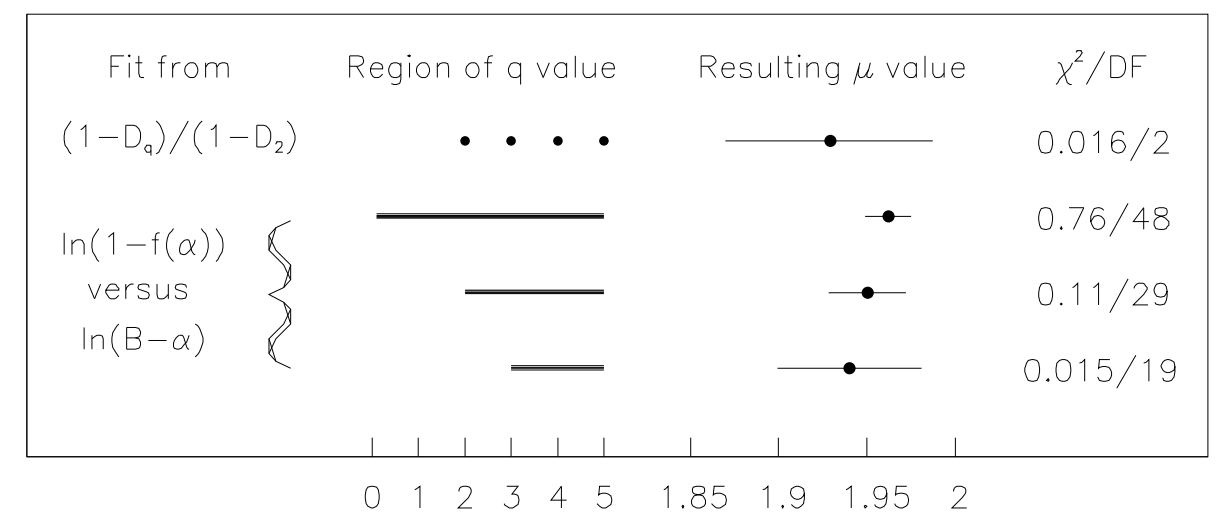

Fig.4 The resulting $\mu$ value from different ways of fitting together with the $q$ region used and the $\chi^{2} / \mathrm{DF}$ 